\title{
A Real-World Analysis of Patients with Untreated Metastatic Epidermal Growth Factor Receptor (EGFR)- Mutated Lung Adenocarcinoma Receiving First-Line Erlotinib and Bevacizumab Combination Therapy
}

\author{
Chin-Chou Wang · Li-Chung Chiu · Pi-Hung Tung · Scott Chih-Hsi Kuo • \\ Chia-Hsun Chu • Allen Chung-Cheng Huang • Chih-Liang Wang • \\ Chih-Hung Chen · Cheng-Ta Yang · Ping-Chih Hsu (D)
}

Received: March 10, 2021 / Accepted: April 16, 2021 / Published online: May 15, 2021

(C) The Author(s) 2021

\section{ABSTRACT}

Introduction: The clinical features of patients with metastatic epidermal growth factor receptor (EGFR)-mutated lung adenocarcinoma

Supplementary Information The online version contains supplementary material available at https:// doi.org/10.1007/s40487-021-00152-6.

C.-C. Wang · C.-L. Wang

Division of Pulmonary and Critical Care Medicine, Kaohsiung Chang Gung Memorial Hospital,

Kaohsiung City 83301, Taiwan

L.-C. Chiu - P.-H. Tung - S. C.-H. Kuo - C.-H. Chu ·

A. C.-C. Huang · C.-H. Chen · C.-T. Yang

P.-C. Hsu $(\square)$

Department of Thoracic Medicine, Chang Gung Memorial Hospital at Linkou, No. 5, Fuxing 1st Rd., Guishan Dist., Taoyuan City 333005, Taiwan e-mail: 8902049@gmail.com;

8902049@adm.cgmh.org.tw

L.-C. Chiu · P.-C. Hsu

Department of Thoracic Medicine, New Taipei

Municipal Tu Cheng Hospital, Chang Gung

Memorial Hospital and Chang Gung University,

New Taipei City 23652, Taiwan

C.-T. Yang

Department of Internal Medicine, Taoyuan Chang

Gung Memorial Hospital, Taoyuan City 33378,

Taiwan

\section{C.-T. Yang}

Department of Respiratory Therapy, College of Medicine, Chang Gung University, Taoyuan City 33302, Taiwan receiving first-line therapy based on erlotinib combined with bevacizumab are unclear. Here, we sought to analyze the clinical features of this patient group.

Methods: Data were analyzed for the period from January 2015 to August 2019 for 49 patients with metastatic EGFR-mutated lung adenocarcinoma receiving first-line erlotiniband-bevacizumab combination therapy from the Linkou and Kaohsiung Chang Gung Memorial Hospitals.

Results: The combination of erlotinib and bevacizumab showed an $83.7 \%$ objective response rate and a $97.9 \%$ disease control rate. The median progression-free survival (PFS) and overall survival (OS) were $22.0 \quad[95 \%$ CI (19.7-22.33)] and 47.6 [95\% CI (38.87-56.37)] months, respectively, for all patients. The secondary EGFR-T790M mutation rate in the patients with acquired resistance to the combination was $72.4 \%$. No predictive factor associated with the appearance of secondary EGFRT790M mutations was found. The most frequent adverse event (AE) caused by the combination therapy was dermatitis $(100 \%)$, and most of the AEs were manageable and grades 1 and 2 . Conclusion: Erlotinib combined with bevacizumab is an effective and safe therapy for untreated metastatic EGFR-mutated lung adenocarcinoma. The combination does not alter secondary EGFR-T790M mutations in patients with acquired resistance and is feasible in realworld clinical practice. 


\section{Graphic Abstract:}

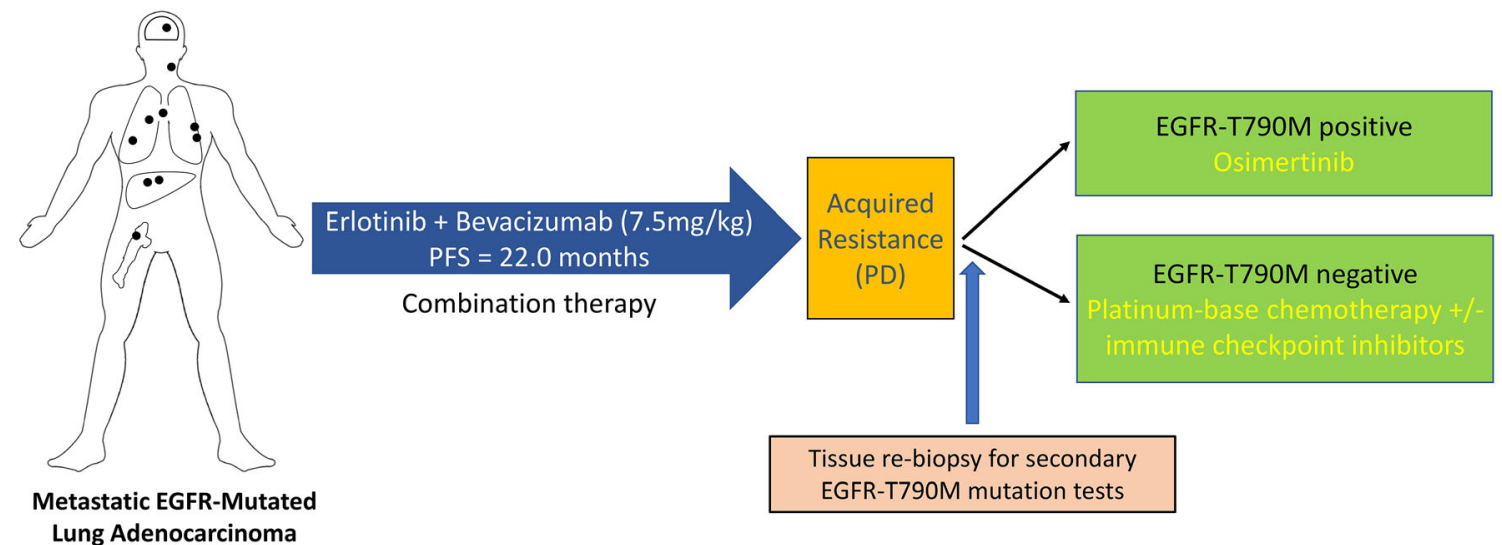

Keywords: Antiangiogenesis; Bevacizumab; Epidermal growth factor receptor mutation; Erlotinib; Lung adenocarcinoma; Tyrosine kinase inhibitor; T790M

\section{Key Summary Points}

\section{Why carry out this study?}

Although the combination of erlotinib and bevacizumab therapy has been approved to treat patients with metastatic EGFR-mutated lung adenocarcinoma based on the results of clinical trials, the experience of using this combination in real-world practice is rarely reported.

The clinical features of patients with metastatic EGFR-mutated lung adenocarcinoma receiving first-line erlotinib combined with bevacizumab therapy are unclear, especially regarding whether the EGFR-T790M mutation is the main cause of acquired resistance to this combination.

\section{What was learned from the study?}

The combination of erlotinib and bevacizumab for untreated metastatic EGFR-mutated lung adenocarcinoma patients in real-world practice is effective, and the toxicities of this combination are manageable and acceptable.

The EGFR-T790M mutation remains the most common cause of acquired resistance to this combination therapy, and most patients receive osimertinib as subsequent therapy.

\section{DIGITAL FEATURES}

This article is published with digital features, including a summary slide, to facilitate understanding of the article. To view digital features for this article go to: https://doi.org/10.6084/ m9.figshare.14423984. 


\section{INTRODUCTION}

Lung adenocarcinoma accounts for most histological types of primary lung cancer (approximately $40 \%$ ), and metastatic disease is present in many patients at the time of initial diagnosis $[1,2]$. An activating epidermal growth factor receptor (EGFR) mutation is the most frequent oncogenic driver mutation in East Asian lung adenocarcinoma patients (ranging from 45 to $55 \%)[3,4]$. Drug therapies targeting activating EGFR mutations, including exon 19 deletion and L858R (a leucine-to-arginine substitution at codon 858: a point mutation in exon 21), have been developed and approved for the treatment of metastatic EGFR-mutated non-small cell lung cancer (NSCLC) patients [4-7]. Erlotinib is classified as a first-generation EGFR-tyrosine kinase inhibitor (TKI) and the first EGFR-TKI approved by the US Food and Drug Administration (FDA) for the treatment of NSCLC following a pivotal clinical trial, BR.21 [4-8]. Erlotinib has also been used as a standard firstline therapy for advanced NSCLC patients harboring exon 19 deletions and L858R mutations because of its promising efficacy, as shown by previous clinical trials (EURTAC and OPTIMAL) $[9,10]$. Two pivotal clinical trials demonstrated that erlotinib showed a $60-80 \%$ objective response rate and a median progression-free survival (PFS) of 10-13 months in treating advanced EGFR-mutated NSCLC patients $[9,10]$.

Pathological angiogenesis is crucial for tumorigenesis in various cancers, and drugs targeting vascular endothelial growth factor (VEGF) and vascular endothelial growth factor receptor (VEGFR) have been developed for anticancer therapy in NSCLC [10, 11]. Bevacizumab is a humanized monoclonal antibody that targets VEGF and is used clinically as a VEGF inhibitor [10, 11]. Two previous clinical trials (E4599 and AVAIL) showed that bevacizumab improved the survival of patients with metastatic non-squamous lung cancer when used in combination with chemotherapy $[12,13]$. Therefore, bevacizumab is the first antiVEGF inhibitor approved for treating advanced non-squamous lung cancer patients [10-13].
The combination of bevacizumab and erlotinib in EGFR-mutated advanced NSCLC patients has been investigated in two previous clinical trials (JO25567 and NEJ026) [14, 15]; both showed that the combination resulted in significantly longer PFS than erlotinib alone in patients with advanced EGFR-mutated NSCLC [14, 15]. Although the two previous trials demonstrated that this combination is an effective therapy, real-world clinical experience using the combination of erlotinib and bevacizumab as first-line therapy in EGFR-mutated NSCLC has seldom been reported. In addition, whether the EGFRT790M mutation is the main cause of acquired resistance in metastatic EGFR-mutated lung adenocarcinoma patients receiving this combination as first-line therapy is still unclear.

In this study, we conducted a real-world multicenter observational study to analyze the clinical features of patients with metastatic epidermal growth factor receptor (EGFR)-mutated lung adenocarcinoma who received erlotinib in combination with bevacizumab as firstline therapy.

\section{METHODS}

\section{Ethics Approval}

This multicenter cohort study was approved by the institutional review board (IRB) (no. 202100379B0) of the Chang Gung Medical Foundation. All study subjects were retrospectively retrieved from the database of Linkou and Kaohsiung Chang-Gung Memorial Hospital (CGMH) Cancer Center. All the patients in this study received standard care and treatment according to the protocol of the Linkou and Kaohsiung CGMH Cancer Center. The IRB of Chang-Gung Medical Foundation waived the need to obtain consent for participation because of the retrospective nature of this study. This study was performed in accordance with the Declaration of Helsinki. We confirmed that there is no identifiable information, such as personal ID or birthdays, reported in this manuscript. 


\section{Patients and EGFR Mutation Tests}

Data were screened on 1018 patients with histologically diagnosed metastatic lung adenocarcinoma with EGFR mutations registered in the Linkou and Kaohsiung CGMH from January 2015 to August 2019. The inclusion criteria were as follows: (1) patients with L858R or exon-19deletion EGFR mutations; (2) patients previously not treated with chemotherapy, targeted therapy, or immunotherapy; (3) patients who did not have previous surgery for primary lung tumor resection; (4) patients who received firstline therapy with erlotinib plus bevacizumab; and (5) patients who had received at least three cycles of bevacizumab during erlotinib therapy. The exclusion criteria were as follows: (1) de novo EGFR-T790M-resistant mutations; (2) patients previously treated with chemotherapy, targeted therapy, or immunotherapy; (3) patients who previously received surgery for primary lung tumor resection; (4) bevacizumab not administered in an erlotinib therapy course; and (5) patients who received fewer than three cycles of bevacizumab during erlotinib therapy. A schematic diagram of the inclusion and exclusion criteria for retrieving the data of the study patients is shown in Fig. 1 .

The clinical medical history, tumor genomic mutations (EGFR mutations), treatment information, and treatment-related adverse effects were retrospectively retrieved from the electronic medical records of Chang Gung Memorial Hospital $(\mathrm{CGMH})$. Each patient underwent computed tomography (CT), positron emission tomography (PET), and brain magnetic resonance imaging (MRI) scans at baseline to determine the stages according to the protocol of the CGMH cancer center. All patients received whole-body CT scans regularly during erlotinib-plus-bevacizumab combination therapy to evaluate treatment response and disease status. Additional images, including chest plain films, sonograms, PET scans, and MRI, were obtained during treatment according to the judgment and needs of the clinical physicians. The last follow-up time point in this study was November 2020.

EGFR mutations were detected by procedures including direct sequencing, amplified refractory mutation system-Scorpion (ARMS/S) assays, and next-generation sequencing (NGS), depending on the physicians' instructions. In

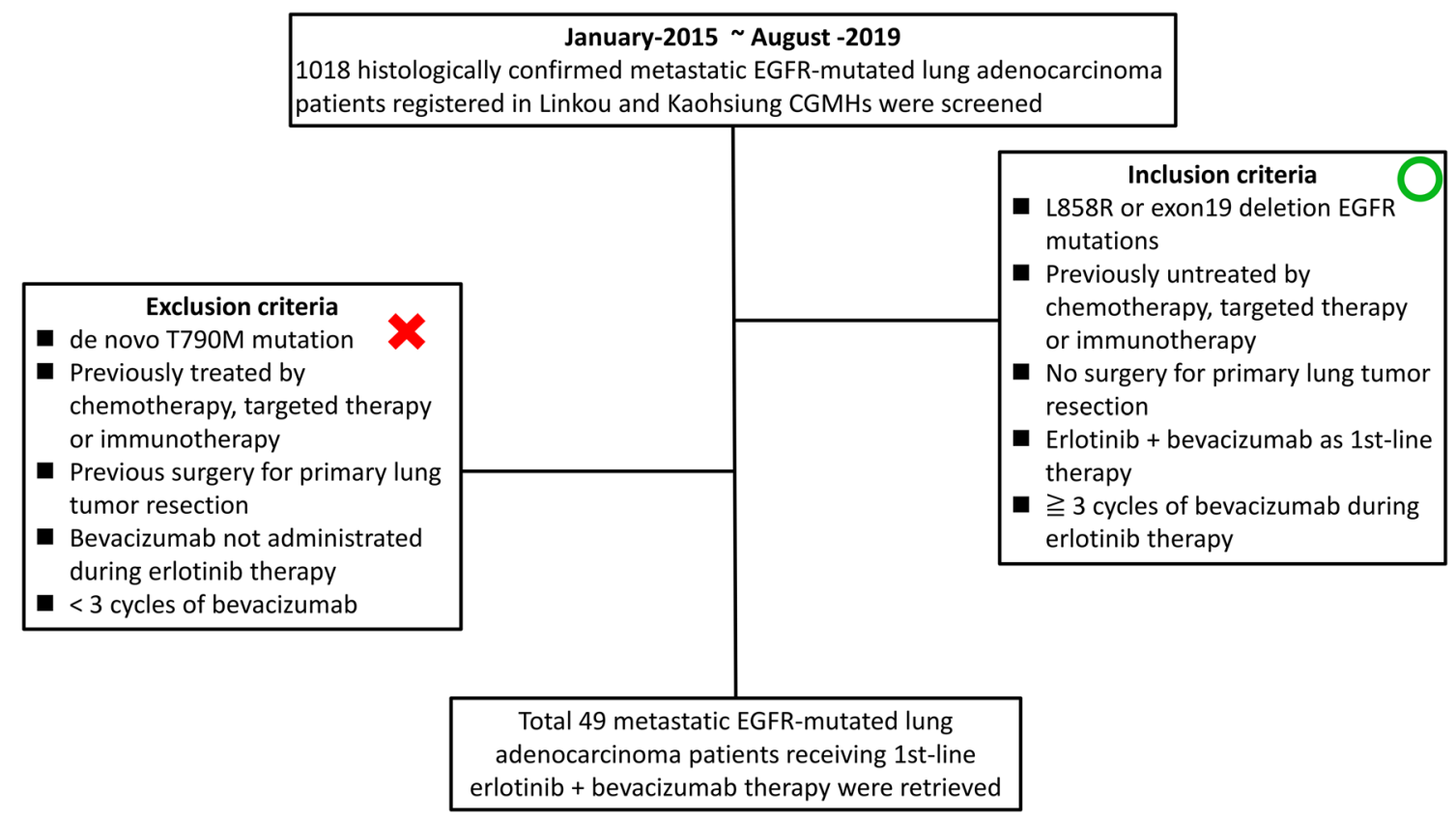

Fig. 1 Scheme of the inclusion and exclusion criteria in this study 
patients who received tumor rebiopsy after the appearance of acquired resistance to erlotinibplus-bevacizumab combination therapy, ARMS/ $\mathrm{S}$ and NGS were used to detect EGFR-T790M mutations.

\section{Evaluation of Efficacy and Treatment- Related Adverse Events (AEs) of Combined Erlotinib-and-Bevacizumab Therapy}

All the patients in this study received erlotinib at a starting dose of $150 \mathrm{mg}$ once daily until disease progression occurred. Bevacizumab was administered intravenously at a dose of $7.5 \mathrm{mg} /$ $\mathrm{kg}$ during erlotinib therapy. The dose and schedule for administering erlotinib and bevacizumab were adjusted by the physicians depending on the patients' clinical condition and treatment-related toxicities. The Response Evaluation Criteria in Solid Tumors (RECIST) version 1.1 was used to assess the treatment response, defined as complete response (CR), partial response (PR), stable disease (SD), or progressive disease (PD). Progression-free survival (PFS) was defined as the time from the first erlotinib dosing date until the date of the first PD images, death, or last follow-up. Overall survival (OS) was defined as the time from the date of diagnosis until the recorded date of death. If patients were still alive at the last follow-up time point (November 30, 2020), survival was censored at the date of the last visit recorded by the physicians.

Treatment-related toxicities and adverse events (AEs) were retrieved retrospectively from the electronic medical records. The grades of AEs were determined according to the National Cancer Institute Common Terminology Criteria for Adverse Events, version 4.0.

\section{Statistical Analysis}

The demographic characteristics and treatment information are presented as quantitative variables. The data for age are presented as the means \pm standard deviations. The frequency distributions between the T790M-positive and T790M-negative groups were tested by Pearson's chi-square test. The rates of survival, including
PFS and OS, were calculated using Kaplan-Meier survival curves. The log-rank test was used for the comparison of survival curves. The $P$ values were considered statistically significant when smaller than 0.05 , and all the $P$ values were twosided. All statistical analyses in this study were performed using GraphPad Prism (version 5.0; GraphPad Software, San Diego, CA, USA).

\section{RESULTS}

\section{Baseline Patient Demographic Characteristics and First-line Treatment Information}

Data for 49 metastatic EGFR-mutated lung adenocarcinoma patients receiving first-line therapy with erlotinib combined with bevacizumab between January 2015 and August 2019 were retrieved and analyzed, and the baseline demographic characteristics and firstline treatment information for all the patients are shown in Table 1 . Among the 49 patients, 17 $(34.7 \%)$ had brain metastasis at initial diagnosis, and seven received radiation therapy to the brain in addition to the combination of erlotinib and bevacizumab. All the patients took erlotinib at a starting dose of $150 \mathrm{mg} /$ day, and six (12.2\%) later had their doses tapered from 150 to $100 \mathrm{mg} /$ day. Regarding the dose of bevacizumab administered, all 49 patients received $7.5 \mathrm{mg} / \mathrm{kg}$ in each cycle. The combination of erlotinib and bevacizumab was discontinued in 33 patients $(67.3 \%)$ at the last follow-up date because of progressive disease but was still ongoing in the other 16 patients $(32.7 \%)$ at the last follow-up.

\section{Efficacy of Erlotinib-Plus-Bevacizumab Combination Therapy: Objective Response, PFS, and OS}

Among the 49 patients in this study, one (2\%) had a CR, and $40(81.6 \%)$ had PRs to the combination of erlotinib and bevacizumab. Seven $(14.3 \%)$ had SD, and one (2\%) had PD at the initial evaluation. The overall objective 
Table 1 Baseline patient characteristics and treatment information of this study

\begin{tabular}{ll}
\hline Variables $($ total $\boldsymbol{n}=\mathbf{4 9})$ & No. \\
\hline Sex & \\
Male & 13 \\
Female & 36 \\
Age (mean \pm SD) & $59.7 \pm$ \\
& 11.1
\end{tabular}

ECOG PS

$\begin{array}{ll}0-1 & 49 \\ \geqq 2 & 0\end{array}$

Smoker

Former + current

Nonsmoker

Histology

Adenocarcinoma

Stage

IV

Brain metastasis at diagnosis

EGFR Mutation

$$
\text { L858R }
$$

Exon 19 deletion

Starting dose of erlotinib

$$
\begin{aligned}
& 150 \mathrm{mg} / \text { day } \\
& 100 \mathrm{mg} / \text { day } \\
& \text { Dose tapering }(150 \mathrm{mg}->100 \mathrm{mg})
\end{aligned}
$$

Bevacizumab dose (each cycle)

$$
7.5 \mathrm{mg} / \mathrm{kg}
$$

Treatment ongoing

Discontinuation because of progressive disease

Local radiation therapy in addition to the combination

\begin{tabular}{ll} 
Brain & 7 \\
Bone & 4 \\
\hline
\end{tabular}

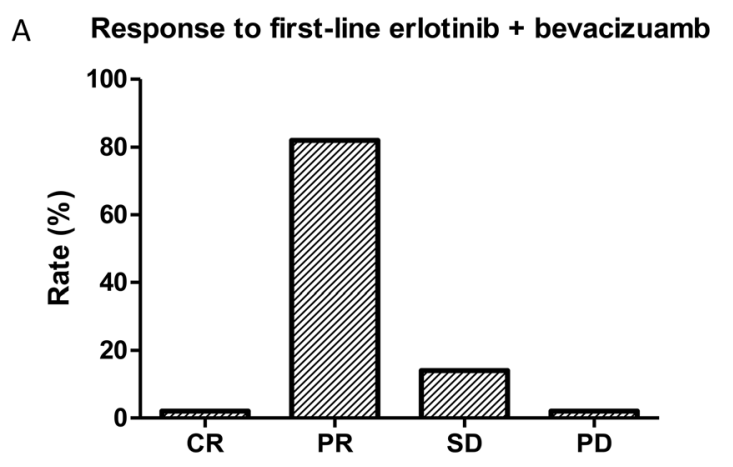

B

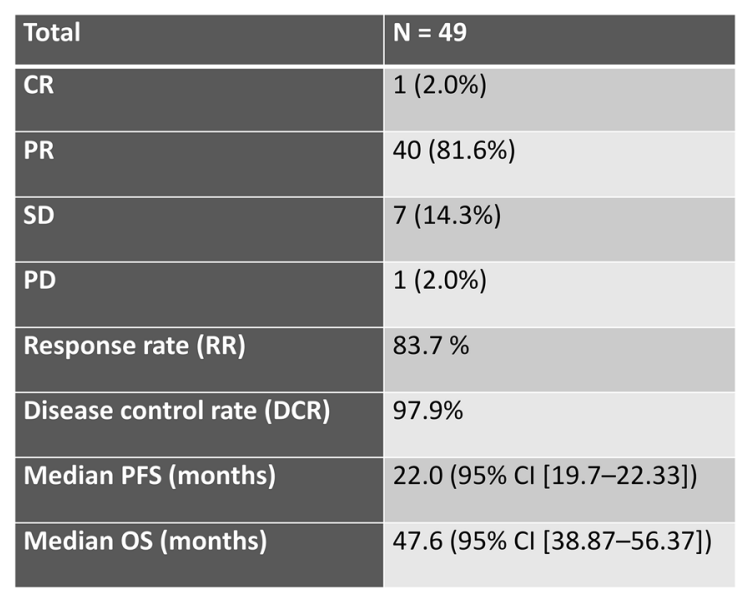

Fig. 2 a Treatment response and (b) efficacy of erlotinib in combination with bevacizumab for untreated metastatic epidermal growth factor receptor (EGFR)-mutated lung adenocarcinoma

response and disease control rates are shown in Fig. 2.

For all 49 patients, the median PFS with erlotinib-plus-bevacizumab combination therapy was 22.0 months (95\% confidence interval (CI) 19.70-22.33 months; Fig. 3a), and the median OS was 47.6 months (95\% CI 38.87-56.37 months; Fig. 3b). When the patients were divided into two groups, namely, L858R and exon-19-deletion mutations, the median PFS with erlotinib combined with bevacizumab was 26.2 and 20.3 months $(95 \%$ CI $0.79-$ 1.79 months, $p=0.586$; Fig. $3 c$ ), respectively, and the median OS was 40.5 and 50.6 months for the L858R-mutation and exon-19-deletion groups (95\% CI $0.46-1.14$ months, $p=0.207$; Fig. 3d), respectively. Between the EGFR-L858R and exon-19-deletion mutated patients, no statistically significant differences were found in 
A

Progression-free survival (PFS) All patients

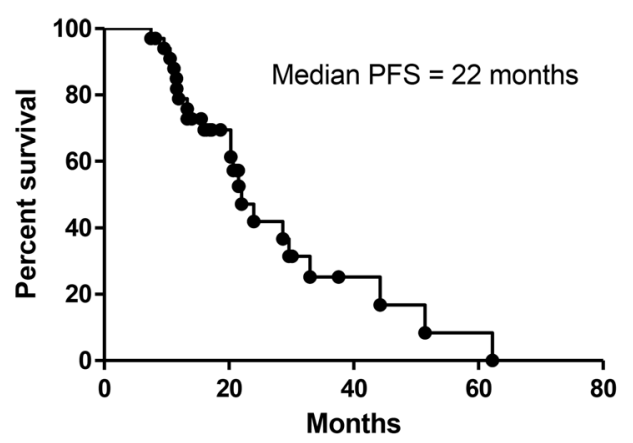

C

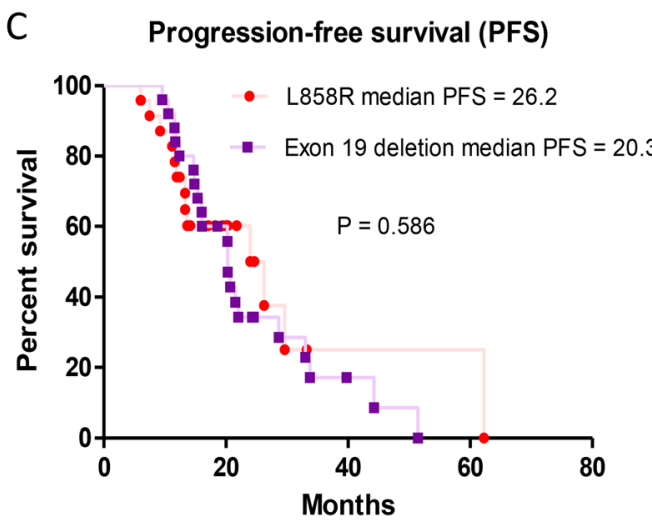

Fig. 3 Kaplan-Meier survival curve of progression-free survival (PFS) and overall survival (OS). a PFS of all patients in this study. $\mathbf{b}$ OS of all patients in this study.

the PFS or OS for the first-line therapy of erlotinib in combination with bevacizumab.

The 17 patients with brain metastasis at initial diagnosis are analyzed and summarized in Supplementary Table S1. The response rate of this combination therapy for the 17 patients was $82.3 \%$. In the 17 patients with brain metastases, the median central nervous system (CNS) PFS and the median OS were 16.1 months (95\% CI 14.1-18.1 months) and 37.4 months (95\% CI 19.6-55.1 months), respectively.

\section{Subsequent Treatments}

after Discontinuation of Erlotinib Combined with Bevacizumab and Secondary EGFR-T790M Mutation

The subsequent treatment information for the 33 patients who discontinued the first-line
B
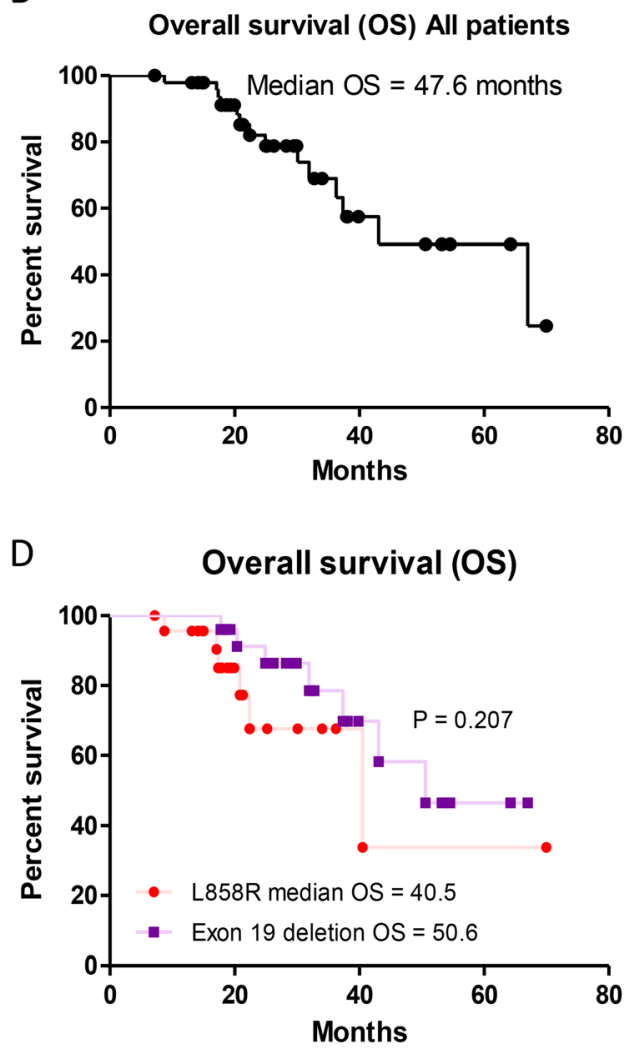

c Comparison of PFS between EGFR-L858R and exon-19deletion mutations. $\mathbf{d}$ Comparison of OS between EGFRL858R and exon-19-deletion mutations

combination of erlotinib and bevacizumab is summarized in Table 2. Among them, 29 underwent tissue rebiopsy for the EGFR-T790M mutation test, and four underwent neither tissue rebiopsy nor blood draw for circulating tumor (ct)-DNA for the EGFR-T790M test. Of the 29 patients who underwent secondary EGFR-T790M tests, 21 had positive results, and eight had negative results for the EGFR-T790M mutation. All 21 patients with EGFR-T790M mutations received the third-generation EGFRTKI osimertinib as subsequent therapy. Three of the patients with EGFR-T790M-mutated tumors received the antiangiogenic agents bevacizumab and ramucirumab in addition to osimertinib. Anti-programmed death 1 (PD-1)/ programmed death-ligand 1 (PD-L1) immunotherapy was subsequently administered in two patients, and one received 
Table 2 Subsequent treatment modalities after discontinuation of the first-line therapy of erlotinib combined with bevacizumab

\begin{tabular}{|c|c|}
\hline Total $n=33$ & No. \\
\hline $\begin{array}{l}\text { EGFR T790M mutation tests (tissue sample re- } \\
\text { biopsy) }\end{array}$ & 29 \\
\hline \multicolumn{2}{|l|}{ EGFR T790M mutation } \\
\hline Positive & 21 \\
\hline Negative & 8 \\
\hline $\begin{array}{l}\text { Unknown (no tissue sample biopsy or ctDNA } \\
\text { for EGFR T790M mutation test) }\end{array}$ & 4 \\
\hline EGFR T790M mutation rate (\%) & $72.4 \%$ \\
\hline \multicolumn{2}{|l|}{ Subsequent therapy } \\
\hline Osimertinib & 21 \\
\hline Platinum-base doublet chemotherapy & 7 \\
\hline Single-agent chemotherapy (pemetrexed) & 1 \\
\hline $\begin{array}{l}\text { Anti-PD-1/PD-L1 immune-checkpoint } \\
\text { inhibitors }\end{array}$ & 2 \\
\hline \multicolumn{2}{|l|}{ Anti-angiogenesis agents } \\
\hline Bevacizumab & 2 \\
\hline Ramucirumab & 2 \\
\hline Supportive care & 3 \\
\hline \multicolumn{2}{|l|}{ Local radiation therapy } \\
\hline Brain & 10 \\
\hline Bone & 5 \\
\hline Liver & 1 \\
\hline
\end{tabular}

ctDNA circulating tumor-DNA

pembrolizumab (anti-PD-1 inhibitor) alone because of strong positive tumor PD-L1 expression (tumor proportion score $>50 \%$ according to the Dako 22c3 assay). The other patients received atezolizumab (an anti-PD-L1 inhibitor) combined with platinum-based chemotherapy. Seven patients received subsequent platinumbase doublet chemotherapy, and one received chemotherapy with pemetrexed alone. One patient received therapy with ramucirumab combined with cisplatin-plus-pemetrexed
Table 3 Comparison of characteristics between T790Mpositive and negative patients with acquired resistance to first-line erlotinib + bevacizumab therapy

\begin{tabular}{|c|c|c|c|}
\hline EGFR T790M mutation & Negative & Positive & $\begin{array}{l}P \text { - } \\
\text { value }\end{array}$ \\
\hline Total & 8 & 21 & \\
\hline Age & & & 0.697 \\
\hline$<60$ & 5 & 11 & \\
\hline$\geq 60$ & 3 & 10 & \\
\hline \multicolumn{4}{|l|}{ Sex } \\
\hline Male & 2 & 6 & 0.961 \\
\hline Female & 6 & 15 & \\
\hline \multicolumn{4}{|l|}{ Smoking status } \\
\hline Nonsmoker & 7 & 15 & 0.666 \\
\hline Former/current smoker & 1 & 6 & \\
\hline EGFR mutation & & & 0.390 \\
\hline L858R & 4 & 6 & \\
\hline Exon 19 deletion & 4 & 15 & \\
\hline Brain metastasis at diagnosis & & & 0.977 \\
\hline Present & 3 & 7 & \\
\hline Absent & 5 & 14 & \\
\hline $\begin{array}{l}\text { Duration of } \\
\text { erlotinib }+ \text { bevacizumab }\end{array}$ & & & 0.209 \\
\hline$<12$ months & 4 & 5 & \\
\hline$\geq 12$ months & 4 & 16 & \\
\hline
\end{tabular}

doublet chemotherapy. Furthermore, 16 patients received palliative local radiation therapy in addition to subsequent second-line therapies, 10 received local radiation therapy to the brain, five received such therapy to the bone, and one received such therapy to the liver.

The 29 patients who underwent tissue rebiopsy tests for the EGFR-T790M mutation were analyzed, and the results are summarized in Table 3. We did not find any statistically significant differences in clinical characteristics 
Table 4 The combination of erlotinib and bevacizumab therapy related adverse events (AEs)

\begin{tabular}{lllll}
\hline Adverse events & All $\boldsymbol{n}=\mathbf{4 9}(\%)$ & Grades 1-2 $\boldsymbol{n}(\%)$ & Grade $\mathbf{3} \boldsymbol{n}(\%)$ & Grade $\mathbf{4} \boldsymbol{n}(\%)$ \\
\hline Dermatitis (skin rash/acne) & $49(100 \%)$ & $42(85.7 \%)$ & $7(14.3 \%)$ & 0 \\
Paronychia & $26(53.1 \%)$ & $26(53.1 \%)$ & 0 & 0 \\
Stomatitis & $23(46.9 \%)$ & $23(46.9 \%)$ & 0 & 0 \\
Diarrhea & $8(16.3 \%)$ & $7(14.3 \%)$ & $1(2.0 \%)$ & 0 \\
Nausea and vomiting & $4(8.2 \%)$ & $4(8.2 \%)$ & 0 & 0 \\
Liver-transaminases increased & $5(10.2 \%)$ & $5(10.2 \%)$ & 0 & 0 \\
Hypertension & $12(24.5 \%)$ & $10(20.4 \%)$ & $2(4.1 \%)$ & 0 \\
Hemorrhage & $3(6.1 \%)$ & $3(6.1 \%)$ & & \\
\hline
\end{tabular}

between the T790M-positive and T790M-negative patients.

\section{Adverse Events (AEs) Related to Therapy with the Combination of Erlotinib and Bevacizumab}

The combination of erlotinib and bevacizumab therapy-related AEs is summarized in Table 4. Among all patients in this study, skin toxicity (100\%) was the most frequent $\mathrm{AE}$, followed by paronychia (53.1\%) and stomatitis (46.9\%). Regarding the side effects of hypertension that could be induced by bevacizumab, 10 patients (20.4\%) experienced grade 1 and 2 hypertension, and two patients had grade 3 hypertension and had to take antihypertensive agents for blood pressure control. Three patients experienced grade 2 hemorrhage, which could be caused by bevacizumab. Among the three patients with hemorrhage, two had bleeding from hemorrhoids, and the other had epistaxis. All bleeding events were stopped by topical medication and skipping one cycle of bevacizumab therapy. There were no serious bleeding complications, such as massive hemoptysis, intracranial hemorrhage or bowel perforation, recorded in this study. Most AEs were manageable by adjusting the erlotinib dose or giving additional drugs, and no patient discontinued the combination therapy because of AEs.

\section{DISCUSSION}

The results of our study provide some important information on untreated metastatic EGFRmutated lung adenocarcinoma patients receiving therapy based on a combination of erlotinib and bevacizumab. This combination is applicable and safe in real-world clinical practice according to the results of this study. First, we demonstrated an objective response rate of $83.7 \%$ and a disease control rate of $97.9 \%$ with this combination. Second, this combination yielded a median PFS of 22.0 months, while the median OS was 47.6 months. Third, the secondary EGFR-T790M mutation rate was $72.4 \%$ in those who experienced acquired resistance to the combination therapy and underwent rebiopsy for EGFR-T790M tests. The most frequent AE was dermatitis, and most of the AEs were limited to grade 1 or 2 , all of which were controllable and reversible.

The use of erlotinib in combination with angiogenesis inhibitors, such as bevacizumab and ramucirumab, as first-line therapy for advanced untreated EGFR-mutated NSCLC has been investigated in previous studies (the JO25567, NEJ026, and RELAY trials) [14-16]. In two previous clinical trials (JO25567 and NEJ026), the efficacy of erlotinib and bevacizumab was shown to be promising, with an objective response rate of $70 \%$ and PFS of approximately 17 months [14, 15]. Ramucirumab is a newer angiogenesis inhibitor, and it is an immunoglobulin that selectively binds 
to VEGFR-2 [10, 11, 17]. Ramucirumab is the second angiogenesis inhibitor approved by the US FDA for use in treating NSCLC based on the results of the REVEL trial [17]. A recent phase III clinical trial (the RELAY trial) showed that the combination of ramucirumab and erlotinib also had excellent efficacy for the treatment of advanced untreated EGFR-mutated NSCLC patients (a 76.6\% objective response rate and 19.4-month PFS) [16]. In our study, 17 patients $(34.7 \%)$ with brain metastasis were recruited, whereas brain-metastatic patients were excluded from the JO25567 and RELAY trials $[14,16]$. In the NEJ026 study, only asymptomatic brainmetastatic patients were included [15]. Brain metastasis actually occurs frequently in advanced EGFR-mutated lung adenocarcinoma (15-30\%), and additional local therapies, including surgery or radiation therapy, are needed in most cases $[18,19]$. In this study, seven patients received radiation therapy for brain metastasis in addition to combination therapy. Previous studies showed that additional bevacizumab therapy might benefit the survival of NSCLC patients with brain metastasis $[20,21]$. Together, these findings may explain why the combination of erlotinib and bevacizumab in our study tended to result in a longer PFS (22.0 months) than in the three clinical studies (the JO25567, NEJ026, and RELAY trials) [14-16].

The economic impact and cost-effectiveness of adding bevacizumab for the treatment of lung cancer are concerns in real-world practice. Insurance policies and coverage are relevant points affecting decisions to add bevacizumab in lung cancer therapy worldwide [22-24]. For example, bevacizumab is not covered by national health insurance for the treatment of lung cancer in Taiwan, and patients receiving bevacizumab have to meet the costs themselves or be covered by additional commercial health insurance $[23,24]$. Based on an analysis of previous clinical studies, bevacizumab at a $7.5 \mathrm{mg} /$ $\mathrm{kg}$ dose is equally effective as a $15 \mathrm{mg} / \mathrm{kg}$ dose when combined with chemotherapy in Asian patients with non-squamous NSCLC $[25,26]$. This is why all the patients in this study received bevacizumab at a $7.5 \mathrm{mg} / \mathrm{kg}$ dose rather than the $15 \mathrm{mg} / \mathrm{kg}$ dose administered in the
JO25567 and NEJ026 trials [14, 15]. Together, the results of our study indicate that erlotinib plus $7.5 \mathrm{mg} / \mathrm{kg}$ bevacizumab, with doses and administration intervals adjustable depending on the physician's judgment of the patient's condition, could be a feasible and effective firstline therapeutic modality for metastatic EGFRmutated lung adenocarcinoma in real-world clinical practice.

There is currently no available predictive biomarker for the usefulness of angiogenesis inhibitors in NSCLC patients, and an earlier study suggested that the addition of bevacizumab in NSCLC patients with high VEGF levels might increase the likelihood of response to the therapy [27]. Two previous studies showed that VEGF expression was higher in EGFR-mutated NSCLC patients than in EGFRwild-type patients $[28,29]$, while two other studies showed that EGFR-mutated NSCLC patients benefited from the addition of bevacizumab to cytotoxic chemotherapy $[29,30]$. Another recent clinical trial, the IMpower150 trial, demonstrated that EGFR-mutated NSCLC patients had significantly improved PFS and OS in the arm receiving a combination therapy regimen including four drugs (bevacizumab + atezolizumab + carboplatin + paclitaxel) [31]. Taken together, these results potentially rationalize the use of bevacizumab in combination with erlotinib for the treatment of patients with untreated metastatic EGFRmutated lung NSCLC either in prospective trials or real-world practice.

The EGFR-T790M point mutation accounts for most of the genomic alterations (30-60\%) leading to secondary acquired resistance in EGFR-mutated NSCLC patients receiving a course of erlotinib therapy [32, 33]. Tissue rebiopsy for EGFR-T790M mutation tests is often required in these patients with acquired resistance to erlotinib therapy $[32,33]$. The third-generation EGFR-TKI osimertinib is an effective subsequent targeted therapy for lung adenocarcinoma patients with secondary EGFRT790M mutations after erlotinib treatment [34]. In the previous three clinical studies (JO25567, NEJ026, and RELAY trials), only a few patients had plasma ctDNA for EGFR-T790M mutation tests (NEJ026), but the data from the tissue 
rebiopsy for secondary EGFR-T790M mutations were unclear [14-16]. In our study, the rate of appearance of secondary EGFR-T790M mutations in the patients who underwent tissue rebiopsy after acquiring resistance to the combination therapy was $72.4 \%$, which was higher than that in NEJO26 (20.8\%). In NEJ026, less than $40 \%$ of patients in the erlotinib plus bevacizumab group had a plasma EGFR-T790M test at the time point of definite progressive disease [35]. A previous study demonstrated that the addition of bevacizumab to EGFR-TKIs induced positive conversion to the T790M mutation in patients with acquired resistance to EGFR-TKIs who were previously negative for T790M-mutated NSCLC [36]. A recent study also showed that the sensitivity of the plasma EGFR test for the secondary T790M mutation in NSCLC with acquired resistance to EGFR-TKIs was low, and tissue rebiopsy was suggested as an appropriate procedure [37]. These findings may explain why the secondary T790M mutation rate in our study was higher than that in the NEJ026 trial. All patients with secondary EGFRT790M mutations took osimertinib in subsequent treatment following first-line erlotinib plus bevacizumab combination therapy. Two previous studies showed that the addition of bevacizumab to the second-generation EGFRTKI afatinib did not decrease the appearance of EGFR-T790M mutations in lung adenocarcinoma patients with acquired resistance to afatinib [36, 38]. Therefore, these results indicate that adding bevacizumab to first- or secondgeneration EGFR-TKIs does not decrease the occurrence of EGFR-T790M mutations in these patients with acquired resistance to erlotinib or afatinib. More than half of the patients in our study were eligible for subsequent osimertinib therapy and indeed took the drug as second-line therapy. Among the 21 patients with EGFRT790M mutations receiving second-line osimertinib in our study, 15 had exon 19 deletions, and 6 had L858R in primary EGFR mutations. The OS analysis of our study showed that exon 19 deletion mutation patients tended to have longer OS than those with the L858R mutation. In the final OS analysis of the NEJ026 trial, patients who received osimertinib as second-line therapy had longer survival than those receiving chemotherapy (50.7 months vs. 40.1 months) [39]. Together, these findings may explain why the OS of patients with the exon 19 deletion mutation was longer than that of patients with the L858R mutation in our study.

The toxicity induced by the combination of erlotinib and bevacizumab should always be kept in mind. Based on the data obtained by previous clinical studies, the increased toxicities frequently induced by adding angiogenesis inhibitors (bevacizumab and ramucirumab) are hypertension, hemorrhage, and neutropenia [10-16, 25, 26]. Angiogenesis inhibitors also increased the skin and gastrointestinal toxicities originally induced by erlotinib [14-16]. Regarding hypertension and bleeding, the occurrence rates were 24.5 and $6.1 \%$, respectively, and they tended to be limited to grades 1 and 2. According to data from previous trials, the rates of hypertension and bleeding in patients treated with angiogenesis inhibitors combined with erlotinib were 40-70 and 20-70\%, respectively [14-16]. Today, clinical physicians are aware of the complications of hypertension and hemorrhage induced by bevacizumab or ramucirumab in previous clinical trials [14-16]. Therefore, bevacizumab administration is avoided in patients with comorbidities such as cardiovascular disease, kidney disease, centrally located tumors with a great vessel and bronchus invasion, cavitary tumors, hemoptysis, and a history of gastrointestinal hemorrhage. This may explain why the rates of hypertension and bleeding recorded in our study tended to be lower than those reported in previous clinical trials [14-16]. Similarly, in a recent clinical observation study, the event rate of hypertension was approximately $20 \%$, and no bleeding events were recorded in NSCLC patients receiving bevacizumab combined with afatinib [38]. The most frequent side effect induced by the combination therapy in this study was skin toxicity, and this was manageable. Only six patients (12.2\%) needed a dose tapering of erlotinib (150-100 mg/day) because of the side effects, but no patient in this study discontinued the combination therapy because of AEs. Our study indicates that the safety of erlotinib in combination with bevacizumab in 
clinical practice was acceptable and that most treatment-related toxicities were manageable.

There are still a few limitations of this study that should be addressed. In real-world clinical practice, the number of patients with metastatic EGFR-mutated lung adenocarcinoma receiving EGFR-TKIs combined with bevacizumab was small, although metastatic EGFR-mutated lung adenocarcinoma patients treated with EGFRTKIs are common in East Asia. The aim of this study was mainly to focus on the feasibility, safety and clinical course of first-line erlotinib combined with bevacizumab therapy for EGFRmutated metastatic lung adenocarcinoma patients in real-world clinical practice, not on the comparison between combination and single-agent therapy. In addition, due to the retrospective nature of this study, we did not recruit suitable control patients with singleagent erlotinib therapy for comparison. The difference in efficacy between erlotinib plus antiangiogenic agents and erlotinib alone can be compared indirectly by reviewing the results of previous studies, including clinical trials and retrospective analyses (Supplementary Table S2) $[9,10,14-16,40-47]$. The median PFS in our study was 22.0 months, and the results of our study suggest that the combination of erlotinib and bevacizumab should be better than singleagent erlotinib therapy in real-world clinical practice. Only East Asian patients were recruited and analyzed in this study; whether this combination has similar efficacy and toxicities in racial groups other than East Asians must be explored in future studies.

\section{CONCLUSION}

The combination of erlotinib and bevacizumab for untreated metastatic EGFR-mutated lung adenocarcinoma patients in real-world practice is effective, and the toxicities of this combination are manageable and acceptable. The combination does not alter the secondary EGFRT790M mutation condition when acquired resistance to the combination therapy develops. More experience-sharing and analysis of the combination of erlotinib and bevacizumab for the treatment of advanced EGFR-mutated
NSCLC patients in clinical practice are warranted in future studies.

\section{ACKNOWLEDGEMENTS}

We thank Ms. Li-Chuan Tsen and Ms. Feng-Ya Shih of the Department of Nursing and Ms. Chiu-Fan Shen for their assistance in retrieving and recording the data of the lung cancer patients. We also thank all other participants of the study. We thank American Journal Experts (AJE) for editorial assistance with the manuscript.

Funding. This study was supported by the Taiwan Ministry of Science and Technology (MOST) (grant no. 109-2628-B-182A-009 to Ping Chih Hsu.) and the Chang-Gung Medical Research Project (grant no. CMRPG3K1561 to Ping Chih Hsu; grant nos. CMRPG8E1661-3, CMRPG8F1441, CMRPG8F1351, CMRPG8H1201, and CORPG8F1491-3 to ChinChou Wang). The Open Access Fee and Rapid Service Fee were funded by the authors.

Authorship. All named authors meet the International Committee of Medical Journal Editors (ICMJE) criteria for authorship for this article, take responsibility for the integrity of the work as a whole, and have given their approval for this version to be published.

Authorship Contributions. Writing of the manuscript: Chin-Chou Wang and Ping Chih $\mathrm{Hsu}$; conception and design of the study: Ping Chih Hsu, Chin-Chou Wang, and Cheng-Ta Yang; acquisition of study data (information of study patients): Ping Chih Hsu, Chin-Chou Wang, Li-Chung Chiu, Pi-Hung Tung, ChihHung Chen, Allen Chung-Cheng Huang, ChihLiang Wang, and Chih-Hung Chen; analysis and interpretation of data: Ping Chih Hsu, LiChung Chiu, Chin-Chou Wang, Scott Chih-Hsi Kuo, Chih-Hung Chen, Pi-Hung Tung, Allen Chung-Cheng Huang, and Cheng-Ta Yang; validation of data: Ping Chih Hsu, Li-Chung Chiu, and Scott Chih-Hsi Kuo; supervision of study work (review and revision of the study): 
Ping Chih Hsu, Scott Chih-Hsi Kuo, and ChengTa Yang; administrative and funding support: Ping Chih Hsu and Chin-Chou Wang.

Disclosures. Chin-Chou Wang, Li-Chung Chiu, Pi-Hung Tung, Scott Chih-Hsi Kuo, ChiaHsun Chu, Allen Chung-Cheng Huang, ChihLiang Wang, Chih-Hung Chen, Cheng-Ta Yang, Ping Chih Hsu have nothing to disclose.

Compliance with Ethics Guidelines. This study was approved by the institutional review board (IRB) (no. 202100379B0) of the Chang Gung Medical Foundation. The IRB of ChangGung Medical Foundation waived the need to obtain consent for participation because of the retrospective nature of this study. All procedures followed were in accordance with the ethical standards of the IRB of Chang-Gung Medical Foundation and with the Helsinki Declaration. No identifiable information, such as personal ID or birthday, was reported in this manuscript.

Data Availability. All data generated or analyzed during this study are included in this published article/as supplementary information files.

Open Access. This article is licensed under a Creative Commons Attribution-NonCommercial 4.0 International License, which permits any non-commercial use, sharing, adaptation, distribution and reproduction in any medium or format, as long as you give appropriate credit to the original author(s) and the source, provide a link to the Creative Commons licence, and indicate if changes were made. The images or other third party material in this article are included in the article's Creative Commons licence, unless indicated otherwise in a credit line to the material. If material is not included in the article's Creative Commons licence and your intended use is not permitted by statutory regulation or exceeds the permitted use, you will need to obtain permission directly from the copyright holder. To view a copy of this licence, visit http://creativecommons.org/licenses/by$\mathrm{nc} / 4.0 /$.

\section{REFERENCES}

1. Goldstraw P, Chansky K, Crowley J, et al. The IASLC lung cancer staging project: proposals for revision of the tnm stage groupings in the forthcoming (Eighth) edition of the TNM classification for lung cancer. J Thorac Oncol. 2016;11(1):39-51.

2. Hsu PC, Miao J, Huang Z, Yang YL, et al. Inhibition of yes-associated protein suppresses brain metastasis of human lung adenocarcinoma in a murine model. J Cell Mol Med. 2018;22:3073-85.

3. Ha SY, Choi SJ, Cho JH, et al. Lung cancer in neversmoker Asian females is driven by oncogenic mutations, most often involving EGFR. Oncotarget. $2015 ; 6: 5465-74$.

4. Hsu PC, Jablons DM, Yang CT, You L. Epidermal growth factor receptor (EGFR) Pathway, yes-associated protein (YAP) and the regulation of programmed death-ligand 1 (PD-L1) in non-small cell lung cancer (NSCLC). Int J Mol Sci. 2019;5:3821.

5. Sharma SV, Bell DW, Settleman J, Haber DA. Epidermal growth factor receptor mutations in lung cancer. Nat Rev Cancer. 2007;7:169-81.

6. Paz-Ares L, Soulières D, Moecks J, Bara I, Mok T, Klughammer B. Pooled analysis of clinical outcome for EGFR TKI-treated patients with EGFR mutationpositive NSCLC. J Cell Mol Med. 2014;18:1519-39.

7. Takeda M, Nakagawa K. First- and Second-generation EGFR-TKIs are all replaced to osimertinib in chemo-naive EGFR mutation-positive non-small cell lung cancer? Int J Mol Sci. 2019;20(1):146.

8. Shepherd FA, Rodrigues Pereira J, Ciuleanu T, et al. Erlotinib in previously treated non-small-cell lung cancer. N Engl J Med. 2005;353(2):123-32.

9. Rosell R, Carcereny E, Gervais R, et al. Erlotinib versus standard chemotherapy as first-line treatment for European patients with advanced EGFR mutation-positive non-small-cell lung cancer (EURTAC): A multicentre, open-label, randomised phase 3 trial. Lancet Oncol. 2012;13:239-46.

10. Zhou C, Wu YL, Chen G, et al. Final overall survival results from a randomised, phase III study of erlotinib versus chemotherapy as first-line treatment of EGFR mutation-positive advanced non-small-cell lung cancer (OPTIMAL, CTONG-0802). Ann Oncol. 2015;26:1877-83.

11. Rajabi M, Mousa SA. The role of angiogenesis in cancer treatment. Biomedicines. 2017;21:34. 
12. Manzo A, Montanino A, Carillio G, et al. Angiogenesis Inhibitors in NSCLC. Int $\mathrm{J}$ Mol Sci. 2021;2017:18.

13. Sandler A, Gray R, Perry MC, et al. Paclitaxel-carboplatin alone or with bevacizumab for non-smallcell lung cancer. N Engl J Med. 2006;355:2542-50.

14. Reck M, von Pawel J, Zatloukal P, et al. Phase III trial of cisplatin plus gemcitabine with either placebo or bevacizumab as first-line therapy for non-squamous non- small-cell lung cancer: AVAil. J Clin Oncol. 2009;27:1227-34.

15. Seto T, Kato T, Nishio M, et al. Erlotinib alone or with bevacizumab as first-line therapy in patients with advanced non-squamous non-small-cell lung cancer harbouring EGFR mutations (JO25567): An open-label, randomised, multicentre, phase 2 study. Lancet Oncol. 2014;15:1236-44.

16. Saito H, Fukuhara T, Furuya N, et al. Erlotinib plus bevacizumab versus erlotinib alone in patients with EGFR-positive advanced non-squamous non-smallcell lung cancer (NEJ026): Interim analysis of an open-label, randomised, multicentre, phase 3 trial. Lancet Oncol. 2019;20:625-35.

17. Nakagawa K, Garon EB, Seto T, et al. Ramucirumab plus erlotinib in patients with untreated, EGFRmutated, advanced non-small-cell lung cancer (RELAY): a randomised, double-blind, placebocontrolled, phase 3 trial. Lancet Oncol. 2019;20: 1655-69.

18. Garon EB, Ciuleanu TE, Arrieta O, et al. Ramucirumab plus docetaxel versus placebo plus docetaxel for second-line treatment of stage IV nonsmall-cell lung cancer after disease progression on platinum-based therapy (REVEL): A multicentre, double-blind, randomized phase 3 trial. Lancet. 2014;84:665-73.

19. Yoshida H, Kim YH, Iwatsubo S, et al. Management and outcomes of newly diagnosed non-small cell lung cancer patients with brain metastases: a realworld study in Japan. Oncology. 2020;98(7):460-7.

20. Rybarczyk-Kasiuchnicz A, Ramlau R, Stencel K. Treatment of brain metastases of non-small cell lung carcinoma. Int J Mol Sci. 2021;22(2):593.

21. Kashiwabara K, Fujii S, Tsumura S, Sakamoto K, Semba H. Additional bevacizumab in EGFR-mutant lung adenocarcinoma patients who had oligo-progression after the failure of EGFR-TKI: a single-institute retrospective study. Cancer Treat Res Commun. 2020;22:100163.

22. Sato $H$, Nagashima $H$, Akiyama $M$, et al. Analysis of bevacizumab treatments and metastatic sites of lung cancer. Cancer Treat Res Commun. 2021;26: 100290.

23. Bittoni MA, Arunachalam A, Li H, et al. Real-world treatment patterns, overall survival, and occurrence and costs of adverse events associated with first-line therapies for medicare patients 65 years and older with advanced non-small-cell lung cancer: a retrospective study. Clin Lung Cancer. 2018;19(5): e629-45.

24. Ahn MJ, Tsai CM, Hsia TC, et al. Cost-effectiveness of bevacizumab-based therapy versus cisplatin plus pemetrexed for the first-line treatment of advanced non-squamous NSCLC in Korea and Taiwan. Asia Pac J Clin Oncol. 2011;7:22-33.

25. Chien CR, Shih YC. Economic evaluation of bevacizumab in the treatment of non-small cell lung cancer (NSCLC). Clinicoecon Outcomes Res. 2012;4:201-8.

26. Reck M, von Pawel J, Zatloukal P, et al. Overall survival with cisplatin-gemcitabine and bevacizumab or placebo as first-line therapy for nonsquamous non-small-cell lung cancer: results from a randomised phase III trial (AVAiL). Ann Oncol. 2010;21:1804-9.

27. Mok TS, Hsia TC, Tsai CM, et al. Efficacy of bevacizumab with cisplatin and gemcitabine in Asian patients with advanced or recurrent non-squamous non-small cell lung cancer who have not received prior chemotherapy: a substudy of the Avastin in Lung trial. Asia Pac J Clin Oncol. 2011;2:4-12.

28. Dowlati A, Gray R, Sandler AB, Schiller JH, Johnson DH. Cell adhesion molecules, vascular endothelial growth factor, and basic fibroblast growth factor in patients with non-small cell lung cancer treated with chemotherapy with or without bevacizumaban Eastern cooperative oncology group study. Clin Cancer Res. 2008;14:1407-12.

29. Reinmuth N, Jauch A, Xu EC, et al. Correlation of EGFR mutations with chromosomal alterations and expression of EGFR, ErbB3 and VEGF in tumor samples of lung adenocarcinoma patients. Lung Cancer. 2008;62:193-201.

30. Tanaka I, Morise M, Miyazawa A, et al. Potential Benefits of Bevacizumab Combined With PlatinumBased Chemotherapy in Advanced Non-Small-Cell Lung Cancer Patients With EGFR Mutation. Clin Lung Cancer. 2020;21(273-280):e4.

31. Jiang Z, Zhang Y, Yang Y, Yue Z, Pan Z. Efficacy of pemetrexed and carboplatin with or without bevacizumab in lung adenocarcinoma patients with EGFR non-T790M mutations after progression on first-line EGFR-tyrosine kinase inhibitors. Thorac Cancer. 2018;9:1151-5. 
32. Reck M, Mok TSK, Nishio M, et al. Atezolizumab plus bevacizumab and chemotherapy in non-smallcell lung cancer (IMpower150): Key subgroup analyses of patients with EGFR mutations or baseline liver metastases in a randomised, open-label phase 3 trial. Lancet Respir Med. 2019;7:387-401.

33. Wu S, Shi X, Si X, et al. EGFR T790M detection in formalin-fixed paraffin-embedded tissues of patients with lung cancer using RNA-based in situ hybridization: A preliminary feasibility study. Thorac Cancer. 2019;10(10):1936-44.

34. Kobayashi N, Katakura S, Kamimaki C, et al. Resistance mechanisms of epidermal growth factor receptor tyrosine kinase inhibitors in non-small cell lung cancer patients: A meta-analysis. Thorac Cancer. 2021 Feb 9.

35. Mok TS, Wu YL, Ahn MJ, et al. Osimertinib or platinum-pemetrexed in EGFR T790M-positive lung cancer. N Engl J Med. 2017;376:629-40.

36. Fukuhara T, Saito H, Furuya N, et al. Evaluation of plasma EGFR mutation as an early predictor of response of erlotinib plus bevacizumab treatment in the NEJ026 study. EBioMedicine. 2020;57: 102861.

37. Hata A, Katakami N, Kaji R, et al. Does afatinib plus bevacizumab combination therapy induce positive conversion of T790M in previously-negative patients? Oncotarget. 2018;9:34765-71.

38. Kim H, Jung HA, Lee $\mathrm{SH}$, et al. Comprehensive evaluation of the clinical utility of plasma EGFR test in non-small cell lung cancer patients with acquired resistance to first-line EGFR inhibitors. Transl Lung Cancer Res. 2021;10(2):878-88.

39. Hsu PC, Huang CY, Wang CC, et al. The combination of afatinib and bevacizumab in untreated EGFR-mutated advanced lung adenocarcinoma: a multicenter observational study. Pharmaceuticals (Basel). 2020;13(11):331.
40. Maemondo M, Fukuhara T, Saito H, et al. NEJ026: Final overall survival analysis of bevacizumab plus erlotinib treatment for NSCLC patients harboring activating EGFR-mutations. Journal of Clinical Oncology 2020 38:15_suppl, 9506-9506

41. Wu YL, Zhou C, Liam CK, et al. First-line erlotinib versus gemcitabine/cisplatin in patients with advanced EGFR mutation-positive non-small-cell lung cancer: analyses from the phase III, randomized, open-label. ENSURE Study Ann Oncol. 2015;26(9):1883-9.

42. Yang JJ, Zhou Q, Yan HH, et al. A phase III randomised controlled trial of erlotinib vs gefitinib in advanced non-small cell lung cancer with EGFR mutations. Br J Cancer. 2017;116(5):568-74.

43. Kuan FC, Li SH, Wang CL, Lin MH, Tsai YH, Yang CT. Analysis of progression-free survival of first-line tyrosine kinase inhibitors in patients with nonsmall cell lung cancer harboring leu858Arg or exon 19 deletions. Oncotarget. 2017;8:1343-53.

44. Tu CY, Chen CM, Liao WC, et al. Comparison of the effects of the three major tyrosine kinase inhibitors as first-line therapy for non-small-cell lung cancer harboring epidermal growth factor receptor mutations. Oncotarget. 2018;9:24237-47.

45. Su VY, Yang KY, Huang TY, et al. The efficacy of first-line tyrosine kinase inhibitors combined with co-medications in Asian patients with EGFR mutation non-small cell lung cancer. Sci Rep. 2020;10(1): 14965.

46. Zhou Q, Wu YL, Cheng Y, et al. 14800 - CTONG 1509: Phase III study of bevacizumab with or without erlotinib in untreated Chinese patients with advanced EGFR-mutated NSCLC, Annals of Oncology, Volume 30, Supplement 5

47. Rosell R, Dafni U, Felip E, et al. Erlotinib and bevacizumab in patients with advanced non-smallcell lung cancer and activating EGFR mutations (BELIEF): an international, multicentre, single-arm, phase 2 trial. Lancet Respir Med. 2017;5(5):435-44. 